\title{
Dissolution Behavior and Thermodynamic Stability of Fused-Sugar Dispersions of a Poorly Water-Soluble Drug
}

\author{
Ghanshyam Singh, Gulshan Chhabra, and Kamla Pathak* \\ Department of Pharmaceutics, Rajiv Academy for Pharmacy, N.H.\#2, P.O. Chattikara, Mathura Uttar Pradesh, India-281001
}

\begin{abstract}
The current research was undertaken to develop and evaluate dissolution profiles of thermodynamically stabilized sugar dispersions of the poorly water-soluble, model drug etoricoxib (ETX). Fused-sugar dispersions were formulated using sorbitol, xylitol, and maltose separately, and the binary systems were stabilized by incorporation of the antiplasticizing agents poloxamer 407 and PVP K30 to obtain ternary and quaternary systems. The sugar dispersions (F1-F12) were subjected to in vitro dissolution studies, and based on model-independent dissolution parameters, ETX-sorbitol binary/ ternary/composite systems were selected for thermodynamic stability study. The aged ETX-sorbitol composite dispersion (SF8) displayed the highest percent dissolution efficiency $\left(\% D E_{80 \min }\right.$ of 78.48$)$, the minimum $t_{50 \%}$ of 4.45 min, and the least tendency to recrystallize $\left(D_{\text {cryst }}\right.$ of 0.68$)$, confirming maximum amorphization and thermodynamic stability among all the composite dispersions. The SEM photomicrographs reveal complete miscibility of drug in the aged quaternary dispersion SF8, and its diffuse reflectance (DR) spectrum confirms the absence of any chemical change. Conclusively, sorbitol binary systems can be effectively stabilized by incorporation of PVP K30-poloxamer 407 and retain the dissolution characteristics of sugar dispersions upon aging.
\end{abstract}

\section{INTRODUCTION}

$\mathrm{D}$ rug release is a crucial and rate-limiting step for oral bioavailability, particularly for drugs with low gastrointestinal solubility and high permeability (1). Many researchers have concluded that an improvement in the release profiles of these drugs by suitable formulation can enhance their bioavailability and reduce side effects $(2,3)$. Class II drugs are considered the best candidates for intervention by formulation, and several methods have been developed to overcome their low aqueous solubility. Salt formation, $\mathrm{pH}$ adjustment, and complexation are wellknown strategies for solubility enhancement, though their use is restricted to a considerably small group of molecules $(4,5)$. Stupak and Bates $(6)$ have reported other ways to improve drug dissolution in aqueous media that include particle size reduction and the addition of solubilizing agents that can promote wettability and increase drug saturation solubility locally. The latter strategies are combined in the formulation of solid dispersions.

Literature is also available on improvement in solubility of BCS Class II drugs by solid dispersion techniques. Reports (7-11) on solid dispersions have conclded that the dissolution properties and hence the bioavailability of slightly water-soluble drug compounds are improved by dispersing them into inert hydrophilic carriers in the solid state. In a solid dispersion, either the drug is dissolved in a matrix or it can be dispersed homogeneously as fine particles in amorphous or crystalline hydrophilic carriers. Various carriers have been used to prepare solid dispersions, and

${ }^{*}$ Corresponding author. sugars are one such carrier. The sugars investigated for the preparation of solid dispersions include lactose for nitrazepam (12) and sucrose, trehalose, and two oligofructoses (inulin DP11 and inulin DP23 having number-average degrees of polymerization of 11 and 23, respectively) for diazepam (13). Similarly, mannitol, lactose, and galactose have been investigated for carbamazepine (12). D-mannitol and sucroester have also been reported for improvement in the oral absorption of triamterene (14) and canrenone (15), respectively. The sugar solid dispersions in each case resulted in an increase in the dissolution rate of the drug, but the single-sugar systems were not thermodynamically stable. The use of sugar combinations overcame some difficulties previously reported with single-sugar systems. Lloyd et al. (16) reported enhancement in the dissolution rates of hydrocortisone and prednisone utilizing ternary sugar solid dispersion systems in tablet form. The dispersion systems were prepared by the fusion method using 50:50 sucrose/mannitol and $50: 50$ sorbitol/mannitol. Both the dissolution profiles and the stability of the ternary systems were better than those of the binary systems.

In sugar dispersions, the drug-sugar ratio markedly affects the dissolution rate and thermodynamic stability of the system. When the drug load is low, the sugar does not dissolve too fast, and the dissolution rate of the drug is high. However, when the drug load is high, the sugar dissolves faster, and the dissolution rate of drug is slow and incomplete. This occurs because a high concentration of drug results in uncontrolled formation of large drug crystals upon aging, and consequently, due to the crystalline nature and low specific surface area, the drug crystals 
Table 1. Parameters Considered for Selection of Carriers of ETX Sugar Dispersion

\begin{tabular}{|c|c|c|c|c|c|}
\hline Parameter & Xylitol & Sorbitol & Maltose & $\begin{array}{l}\text { Povidone } \\
\text { K30 }\end{array}$ & $\begin{array}{c}\text { Poloxamer } \\
407\end{array}$ \\
\hline Category & Sugar & Sugar & Sugar & $\begin{array}{l}\text { Synthetic } \\
\text { polymer }\end{array}$ & Surfactant \\
\hline $\begin{array}{l}\text { Melting } \\
\text { point }\end{array}$ & $92-96^{\circ} \mathrm{C}$ & $110-112^{\circ} \mathrm{C}$ & $120-125^{\circ} \mathrm{C}$ & $127-135^{\circ} \mathrm{C}$ & $52-57^{\circ} \mathrm{C}$ \\
\hline $\begin{array}{l}\text { Solubility } \\
\text { in water }\end{array}$ & 1 in 1.6 & 1 in 0.5 & 1 in 2.0 & $\begin{array}{l}\text { Freely } \\
\text { soluble }\end{array}$ & $\begin{array}{l}\text { Freely } \\
\text { soluble }\end{array}$ \\
\hline
\end{tabular}

dissolve slowly (12). If the crystallization process of drug in the solid dispersions is retarded or inhibited, the changes in dissolution profiles of sugar dispersions upon aging can be overcome. Attia et al. (12) and Chauhan et al. (17) reported the use of antiplasticizing agents like polyvinylpyrrolidone (PVP K30) that tend to improve the thermodynamic stability of sugar dispersions and consequently minimize changes in dissolution behavior.

To enhance wetting characteristics and solubilization of the drug, surface-active agents are often used in solid dispersions. However, owing to their potential for mucosal irritation, these are preferentially used in combination with other carriers, usually consisting of one or more polymers (18). Besides increasing wettability, a polymer can prolong the existence of a microenvironment in which the solubility of the drug is enhanced due to a high concentration of the carrier in the surrounding solution (2). Thus for a poorly soluble drug, incorporation of surfactant and polymer in a sugar dispersion will have the additive effect of improving both dissolution characteristics and thermodynamic stability.

Etoricoxib, 5-chloro-2-[6-methylpyridine-3-yl]-3-[4methylsulfoxylphenyl] pyridine (ETX), a BCS Class II drug with water solubility of $3.28 \mu \mathrm{g} / \mathrm{mL}$ and a wetting time of 149 min, was selected as a model drug for making sugar dispersions along with agents that can improve the thermodynamic stability upon aging. Another important consideration was its low melting point that supports the use of the fusion method (solvent-free) for the preparation of sugar dispersions. Thus, ETX sugar dispersions were aimed to improve the dissolution of a poorly watersoluble model drug and to evaluate the thermodynamic stability of the aged dispersions upon incorporation of antiplasticizing agent. Selection of carriers for the preparation of thermodynamically stable sugar dispersions was based on their solubility in water and low melting points. The properties of these carriers are compiled in Table 1.

\section{MATERIALS AND METHODS \\ Materials}

Etoricoxib was obtained as a gift sample from Sun Pharmaceuticals Pvt. Ltd., Mumbai, India. Xylitol and PVP K30 were supplied by S.D. Fine Chemicals, Ltd.,
Mumbai, India. Sorbitol and maltose were purchased from Central Drug House Ltd., New Delhi, India, and poloxamer 407 was obtained as a gift sample from BASF Corporation, United States. All chemicals were of analytical grade and were used without further purification.

\section{Preparation of Sugar-Polymeric/Surfactant Dispersions}

The binary/ternary/quaternary sugar dispersions of ETX (F1-F12) were prepared by a fusion method wherein the drug, sugar, and polymer/surfactant (Table 2) were blended thoroughly for 15 min in a glass mortar and pestle and transferred to a porcelain dish. Each mixture was heated carefully in an oil bath (liquid paraffin, $140^{\circ} \mathrm{C}$ ) with constant stirring until a clear homogeneous melt was obtained. The molten mass was quickly transferred into an ice bath and allowed to solidify. After solidification, each mixture was pulverized in a glass mortar and pestle, sifted through a no. $44(355 \mu \mathrm{m})$ sieve, and stored in a desiccator until use.

The quaternary physical mixtures of ETX were prepared by blending requisite quantities of drug with sugar, polymer, and surfactant in a glass mortar and pestle for $15 \mathrm{~min}$ to get homogeneous mixtures that were passed through a no. $44(355 \mu \mathrm{m})$ sieve. The resulting mixtures were stored in desiccators until further use.

\section{In Vitro Dissolution Studies}

In vitro dissolution testing of pure ETX (F0), physical mixtures, and sugar dispersions equivalent to $60 \mathrm{mg}$ of ETX (F1-F12) was carried out using USP Apparatus 2 (paddle) at $50 \mathrm{rpm}$ and $37 \pm 0.5^{\circ} \mathrm{C}$. A powder dispersion method in $\mathrm{pH} 7.4$ phosphate buffer (0.2 M potassium dihydrogen phosphate $\mathrm{KH}_{2} \mathrm{PO}_{4}$ and a specified volume of $0.2 \mathrm{M}$ sodium hydroxide, as per IP1996) was used. Five milliliters of sample was withdrawn at predetermined times and replaced with an equal volume of fresh dissolution medium maintained at $37 \pm 0.5^{\circ} \mathrm{C}$. Each aliquot was filtered through a $0.45-\mu \mathrm{m}$ nylon disc filter, and the absorbance was read at $284 \mathrm{~nm}$ by UV spectrophotometer (PharmaSpec1700, Shimadzu, Tokyo, Japan). The percent of ETX dissolved at various time intervals was calculated. The percent cumulative drug dissolved was plotted versus time, and the profiles were used to deter mine the dissolution enhancement ratio and percent dissolution efficiency. The dissolution enhancement ratio was calculated by dividing the percent cumulative drug dissolved from binary/ternary/quaternary systems by the percent drug dissolved from F0 at 60 min.

Various model-independent parameters (i.e., dissolution efficiency at $50 \mathrm{~min}\left[\% D E_{50 \mathrm{~min}}\right], 80 \mathrm{~min}\left[\% D E_{80 \mathrm{~min}}\right]$, time taken for $50 \%\left[t_{50 \%}\right]$ and $80 \%$ [ $\left.t_{80 \%}\right]$ release) were computed using PCP DISSO v2.08 software, Pune, India. The dissolution efficiency $(D E)$ is the area under the dissolution curve between two time points $t_{1}$ and $t_{2}$ expressed as a percentage of the curve at maximum dissolution (i.e., 100\% dissolved) over the same time period. The dissolution efficiency of the solid complexes was calculated by following equation: 


\begin{tabular}{|c|c|c|c|c|c|}
\hline Formulation Code & Drug (mg) & Sugar (mg) & PVP K30 (mg) & Poloxamer 407 (mg) & $\begin{array}{l}\text { Percent dissolution } \\
\text { enhancement } t=60 \mathrm{~min}\end{array}$ \\
\hline \multicolumn{6}{|l|}{ Xylitol } \\
\hline F1 & 60 & 540 & - & - & 1.98 \\
\hline F2 & 60 & 540 & 60 & - & 2.26 \\
\hline F3 & 60 & 540 & - & 60 & 2.06 \\
\hline F4 & 60 & 540 & 60 & 60 & 2.51 \\
\hline PMF4 & 60 & 540 & 60 & 60 & 1.48 \\
\hline \multicolumn{6}{|l|}{ Sorbitol } \\
\hline F5 & 60 & 540 & - & - & 2.10 \\
\hline F6 & 60 & 540 & 60 & - & 2.42 \\
\hline F7 & 60 & 540 & - & 60 & 2.38 \\
\hline F8 & 60 & 540 & 60 & 60 & 2.57 \\
\hline PMF8 & 60 & 540 & 60 & 60 & 1.82 \\
\hline \multicolumn{6}{|l|}{ Maltose } \\
\hline F9 & 60 & 540 & - & - & 1.74 \\
\hline F10 & 60 & 540 & 60 & - & 2.18 \\
\hline F11 & 60 & 540 & - & 60 & 2.28 \\
\hline F12 & 60 & 540 & 60 & 60 & 2.36 \\
\hline PM12 & 60 & 540 & 60 & 60 & 1.42 \\
\hline
\end{tabular}

${ }^{a}$ At 60 min with respect to ETX dissolution, $F O$.

$\%$ Dissolution Efficiency $(\% D E)=\frac{\int_{0}^{t} y d t}{y 100(t 2-t 1)} \times 100$

\section{Thermodynamic Stability Study}

Based on in vitro drug dissolution data, binary, ternary, and quaternary sugar dispersions F5-F8 were selected for thermodynamic stability study. F4 and F12 were used as reference quaternary sugar dispersions. The selected ETX sugar dispersions were placed in sealed, clear glass vials and stored at $40^{\circ} \mathrm{C}$ and $75 \% \mathrm{RH}$ for a period of two months. At the end of the storage period, the aged samples were evaluated for in vitro dissolution behavior using USP Apparatus 2 (detailed in previous section) and compared with the dissolution profiles of fresh sugar dispersions of ETX. In addition to model-independent dissolution parameters, the dissolution profiles of fresh binary/ternary/quaternary sugar dispersions were compared with those of aged binary/ternary/quaternary sugar dispersions by similarity factors $\left(f_{2}\right)$ calculated using

$$
f_{2}=50 \cdot \log \left\{\left[1+(1 / \mathrm{n}) \sum_{t=1}^{n}\left(R_{t}-T_{t}\right)^{2}\right]^{-0.5} \cdot 100\right\}
$$

where $n$ is the sample number and $R_{t}$ and $T_{t}$ are the percentages of the reference and test drug release, respectively, at different time intervals. If $f_{2}$ of two dissolution release profiles is between 50 and 100, then these drug release profiles are similar. A value under 50 indicates differences between the release profiles (19).

\section{Assessment of Recrystallization in Stored ETX Sugar Dispersions by DSC}

For DSC thermograms, about $10 \mathrm{mg}$ of sample was sealed in an aluminum pan, and an empty aluminum pan was used as reference (Perkin- Elmer, Pyris Diamond, USA). The thermograms were recorded under nitrogen flow (20 $\mathrm{mL} / \mathrm{min}$ ) at a scanning rate of $10^{\circ} \mathrm{C} / \mathrm{min}$ in the range of $30-300^{\circ} \mathrm{C}$. The enthalpy values and the temperature of the endothermic peaks were used to determine the degree of crystallinity $\left(D_{\text {crys }}\right)$ of the drug in the sugar dispersions. $D_{\text {crys }}$ is defined as the ratio of the heat of fusion of the sugar dispersion $\left(\Delta H_{\mathrm{sd}}\right)$ and the heat of fusion of pure drug $\left(\Delta H_{\mathrm{dr}}\right)$ multiplied by the fraction of drug $(f)$ in the dispersion (20).

$$
D_{\text {crys }}=f \Delta H_{\text {sd }} / \Delta H_{\text {dr }} \times 100 \%
$$

\section{Scanning Electron Microscopy}

The morphology of sugar dispersions was characterized by scanning electron microscopy using a LEO 435 Variable 


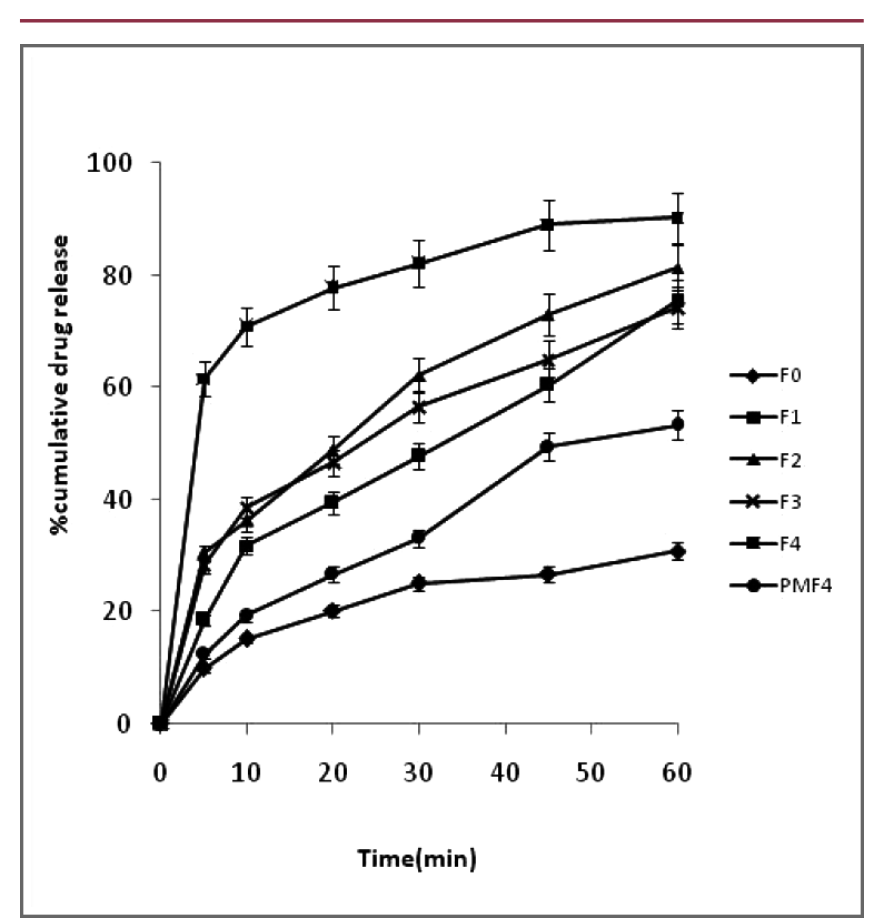

Figure 1. In vitro dissolution profiles of ETX-xylitol dispersions in $\mathrm{pH} 7.4$ phosphate buffer.

Pressure SEM (SEMTech Solutions, Inc., North Billerica, MA). The samples were fixed on a brass stub using doublesided tape and then gold-coated in vacuum by a sputter coater. Samples were then observed with scanning electron microscopy under various magnifications.

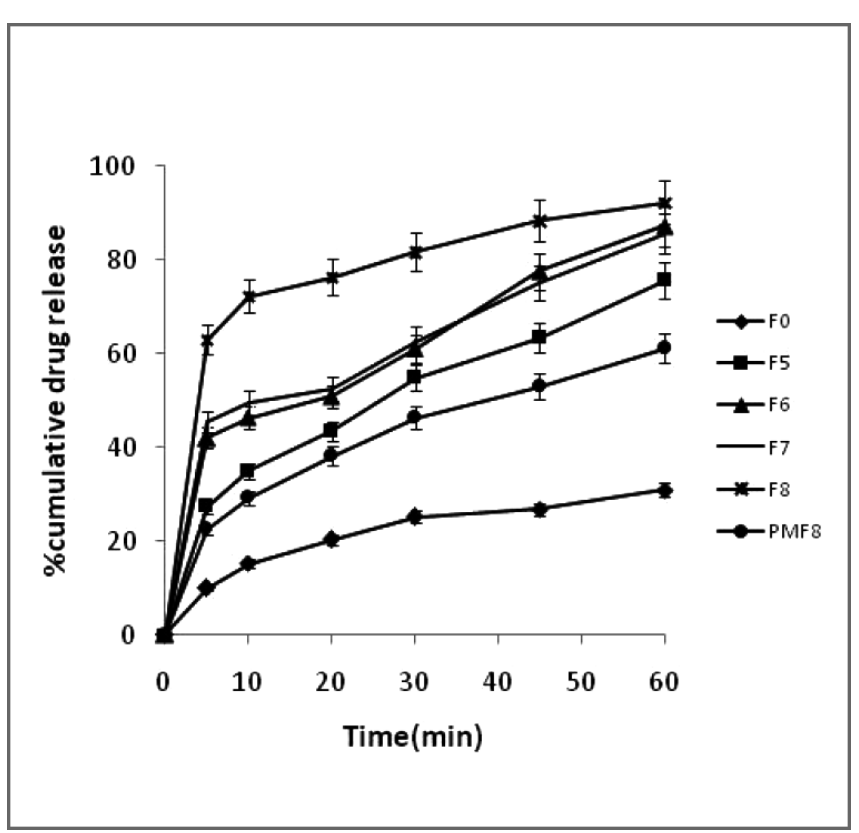

Figure 2. In vitro dissolution profiles of ETX-sorbitol dispersions in $\mathrm{pH} 7.4$ phosphate buffer.

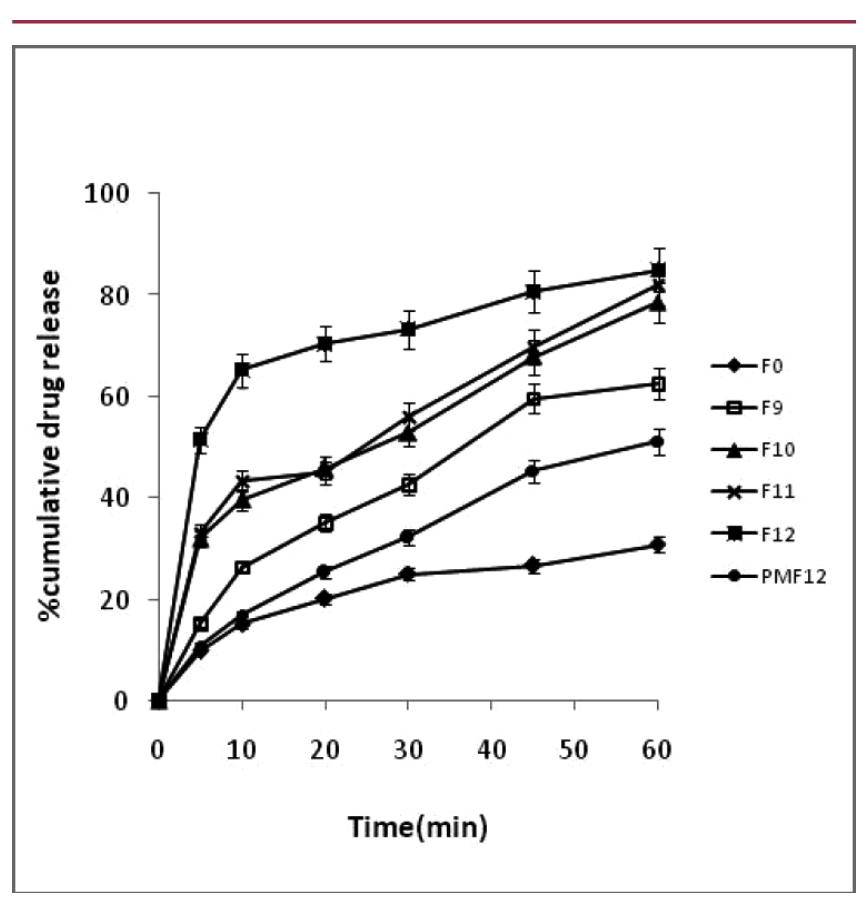

Figure 3. In vitro dissolution profiles of ETX-maltose dispersions in $\mathrm{pH} 7.4$ phosphate buffer.

\section{Diffuse Reflectance Spectroscopy}

Diffuse reflectance spectrographs of the pure drug, sugar, PVP K30, poloxamer 407, physical mixtures, and sugar dispersions were obtained with a Shimadzu FTIR8400 (Kyoto, Japan) using IR grade KBr powder. About 2-3 $\mathrm{mg}$ of the sample was mixed with dry $\mathrm{KBr}$ and scanned over the range $4000-400 \mathrm{~cm}^{-1}$.

\section{RESULTS AND DISCUSSION \\ In Vitro Dissolution Studies}

The in vitro dissolution profiles of the binary/ternary/ quaternary ETX sugar dispersions were compared with those of pure ETX (F0) and respective physical mixtures (Figures 1-3). The profile of pure ETX was characterized by a slow gradual dissolution of $31 \%$ within 60 min in $\mathrm{pH} 7.4$ phosphate buffer. Among the dispersions prepared utilizing three different sugars, dissolution of ETX from sorbitol dispersions was highest within 60 min when compared with xylitol and maltose dispersions. This is because sorbitol has higher solubility than xylitol or maltose in aqueous media and hence dissolves faster, thereby creating a hydrophilic environment that is conducive for dissolution of ETX.Thus, there was a considerable increase in percent dissolution for fused ETX binary/ternary/quaternary dispersions as compared with pure ETX. The order of increasing dissolution was quaternary $>$ ternary $>$ binary, and the quaternary dispersion displayed higher dissolution than quaternary physical mixtures for all the sugars evaluated.

In the binary systems made with drug/sugar (1:9), the enhancement in dissolution was greatest with sorbitol (Table 2) followed by xylitol and maltose, which is 
Table 3. Model-Independent Parameters of In Vitro Dissolution Profiles of Fresh and Aged ${ }^{a}$ Binary/Ternary/Quaternary Sugar Dispersions of ETX

\begin{tabular}{|c|c|c|c|c|c|c|c|c|c|c|}
\hline \multicolumn{2}{|c|}{ Formulation code } & \multicolumn{2}{|c|}{$\% \mathrm{DE}_{50 \min }$} & \multicolumn{2}{|c|}{$\% \mathrm{DE}_{80 \min }$} & \multicolumn{2}{|c|}{$\mathbf{t}_{50 \%}(\min )$} & \multicolumn{2}{|c|}{$\mathbf{t}_{80 \%}(\min )$} & \multirow[b]{2}{*}{ Similarity factor (f2) } \\
\hline Fresh sample & Aged sample & 0 & 2 & 0 & 2 & 0 & 2 & 0 & 2 & \\
\hline $\mathrm{F} 4^{b}$ & $\mathrm{SF} 4^{b}$ & 72.10 & 62.61 & 79.55 & 62.61 & 4.06 & 5.06 & 29.23 & 59.33 & 44.76 \\
\hline F5 & SF5 & 44.66 & 44.26 & 57.04 & 55.09 & 27.41 & 28.63 & 83.37 & 121.22 & 38.44 \\
\hline F6 & SF6 & 56.00 & 48.83 & 67.59 & 60.39 & 19.71 & 20.50 & 46.49 & 116.51 & 42.57 \\
\hline F7 & SF7 & 56.92 & 49.51 & 67.49 & 68.59 & 19.09 & 13.50 & 56.10 & 90.64 & 43.99 \\
\hline F8 & SF8 & 74.89 & 71.45 & 81.66 & 78.48 & 3.97 & 4.45 & 29.44 & 30.48 & 68.91 \\
\hline $\mathrm{F} 12^{b}$ & $\mathrm{SF} 12^{b}$ & 67.50 & 44.59 & 73.94 & 59.89 & 4.85 & 10.96 & 44.59 & 150.22 & 41.22 \\
\hline
\end{tabular}

${ }^{a}$ Aged two months.

${ }^{\circ}$ Reference formulations.

attributable to the higher solubility of sorbitol. Dissolution was further enhanced in ternary dispersions made with PVP K30 and poloxamer 407 separately (Figures 1-3, Table 2). The short swelling time of PVP K30 prior to dissolution and the prevention of nucleation were the contributing factors for the increase in solubility and consequent higher dissolution rate of drug (21) from the ternary dispersion made with PVP K30. On the other hand, the enhancement in dissolution of the ternary dispersion made with poloxamer 407 (1:9:1 ETX/sugar/ antiplasticizing agent) was due to the formation of hydrophilic amorphous product. Because of its oxyethylene content, the surfactant dissolved rapidly in the dissolution medium creating a microenvironment that played a major role in solubility enhancement (22). Though the antiplasticizing agents differed in their mechanisms, the enhancement of dissolution in comparison to pure drug was similar (2.06-2.42\%) for all categories of sugar dispersions (Table 2). For ternary dispersions of xylitol and sorbitol, the effect of PVP K30 on dissolution was more pronounced than that of poloxamer 407, whereas for maltose ternary dispersions, the enhancement in dissolution by surfactant was greater than by PVP K30.

To analyze the additive effect of incorporating both antiplasticizing agents, dissolution of the quaternary fused dispersions was performed, and enhancements of $2.51 \%$, $2.57 \%$, and $2.36 \%$ were documented for xylitol, sorbitol, and maltose quaternary dispersions, respectively. The possible mechanisms of increased dissolution rate of ETX sugar dispersions include reduction in drug particle size, carrier solubilization effect, absence of aggregation of drug crystallites, improved wettability and dispersibility of drug, dissolution of drug in hydrophilic carriers, conversion of drug to the amorphous state, or a combination of these methods (23-25). Because the dissolution enhancement of the quaternary maltose dispersion was less than that of ternary dispersions of the other two sugars, the maltose dispersions were not investigated any further. On the other hand, sorbitol dispersions were selected for thermodynamic stability investigations because the dissolution enhancement values were higher than for those for xylitol.

For a better understanding, the dissolution data of ETX from sugar dispersions were subjected to a model-independent analysis (Table 3). An important model-independent parameter, dissolution efficiency $(D E)$, was computed. Because $D E$ takes into account the dissolution profile as a whole, as opposed to $t_{50 \%}$ values, this approach employs a more realistic and meaningful method of comparing various formulations. It was concluded that dissolution efficiency at $50 \mathrm{~min}$ and $80 \mathrm{~min}$ was highest for quaternary dispersion F8, and the time for $50 \%$ and $80 \%$ drug dissolution was least among all the quaternary dispersions. Thus, based on dissolution analysis, the F8 sugar dispersion was considered the best formulation. Further studies on thermodynamic stability were carried out using the sorbitol dispersions, and quaternary dispersions F4 and F12 were used as reference formulations.

\section{Thermodynamic Stability of Sugar Dispersions}

One of the major problems of sugar dispersions is their thermodynamic instability; upon storage, these undergo transformation from amorphous to crystalline form that can potentially affect the dissolution characteristics. Thus, a formulation made with these dispersions may result in unpredictable drug-release profiles. The addition of polymer or surfactant in sugar dispersions is a significant approach used to overcome this problem.

The protective effect of PVP K30 (26) can be attributed to two properties of the hydrophilic polymer: (1) the antiplasticizing effect that increases the temperature at which the molecular mobility becomes significant with respect to recrystallization, and (2) an improvement in the glass transition temperature $\left(T_{\mathrm{g}}\right)$ of a miscible mixture that decreases the molecular mobility at regular storage temperature. On the other hand, poloxamer 407 creates a 


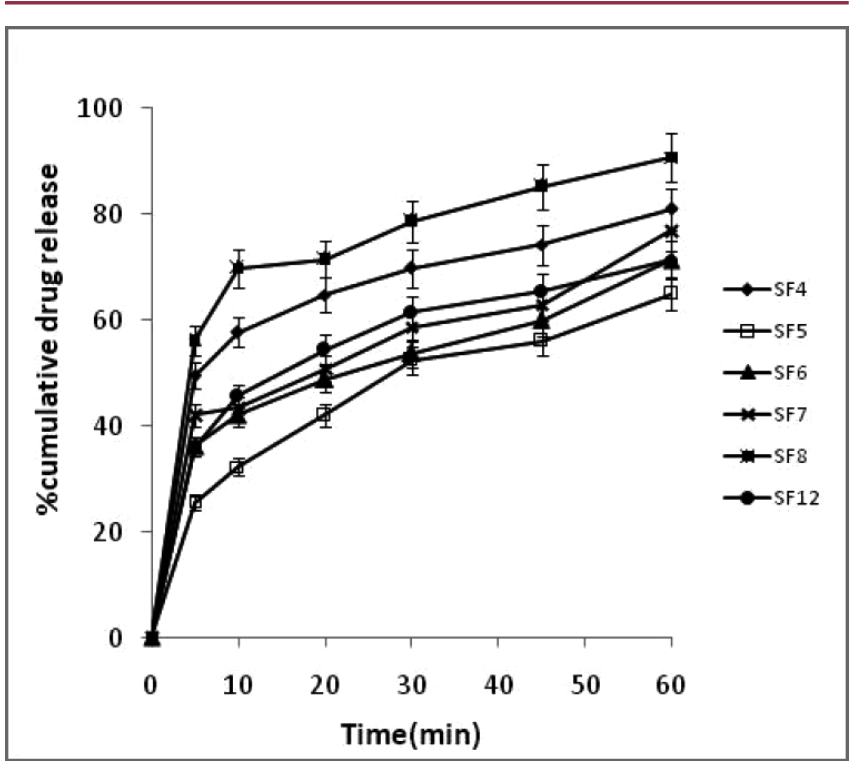

Figure 4. In vitro dissolution profiles of aged ETX-sorbitol dispersions in pH 7.4 phosphate buffer.

microenvironment around the dissolving particles that increases solubility and wetting of growing crystals, thus preventing any transformation. To confirm this concept, the sugar dispersions F4-F8 and F12 were subjected to in vitro dissolution studies after storage at $40{ }^{\circ} \mathrm{C}$ and $75 \%$ $\mathrm{RH}$ for a period of two months. The dissolution profiles of aged samples (Figure 4) were compared with the release profiles of fresh sugar dispersions using the model-independent parameters $\% D E_{50 \min }$ and $\% D E_{80 \min }$ representing the early and late phases of dissolution (Table 3). Maximum \%DE values of $71.45 \%$ at $50 \mathrm{~min}$ and $78.48 \%$ at $80 \mathrm{~min}$ were found for SF8 (aged quaternary dispersion) and minimum values of $44.26 \%$ at $50 \mathrm{~min}$ and $55.09 \%$ at 80 min for SF5 (aged binary dispersion).

Consequently, the times taken for $50 \%$ drug release $(4.45$ $\mathrm{min}$ ) and $80 \%$ drug release (30.48 $\mathrm{min}$ ) were least for the SF8 dispersion and were comparable to the dissolution parameters of the fresh sample F8. This was confirmed by calculation of similarity factor between the release profiles of fresh and aged samples. The value of the similarity factor $\left(f_{2}\right)$ was maximum $(68.91 \%)$ and greater than 50 for SF8 when compared with F8, which implies that dissolution profiles are similar and unchanged; this clearly demonstrates the stabilization effect of antiplasticizing agents in sorbitol dispersion. However, SF4, the aged xylitol dispersion of $\mathrm{F} 4$ that exhibited comparative dissolution enhancement as sorbitol dispersion F8, exhibited a similarity factor of 44.76 when compared with the dissolution profile of fresh dispersion F4. Thus, the antiplasticizing effect was not remarkable in xylitol dispersion, in contrast to sorbitol dispersions that could be thermodynamically stabilized by incorporation of PVP K30 and poloxoamer 407. This clearly indicates the role of antiplasticizing

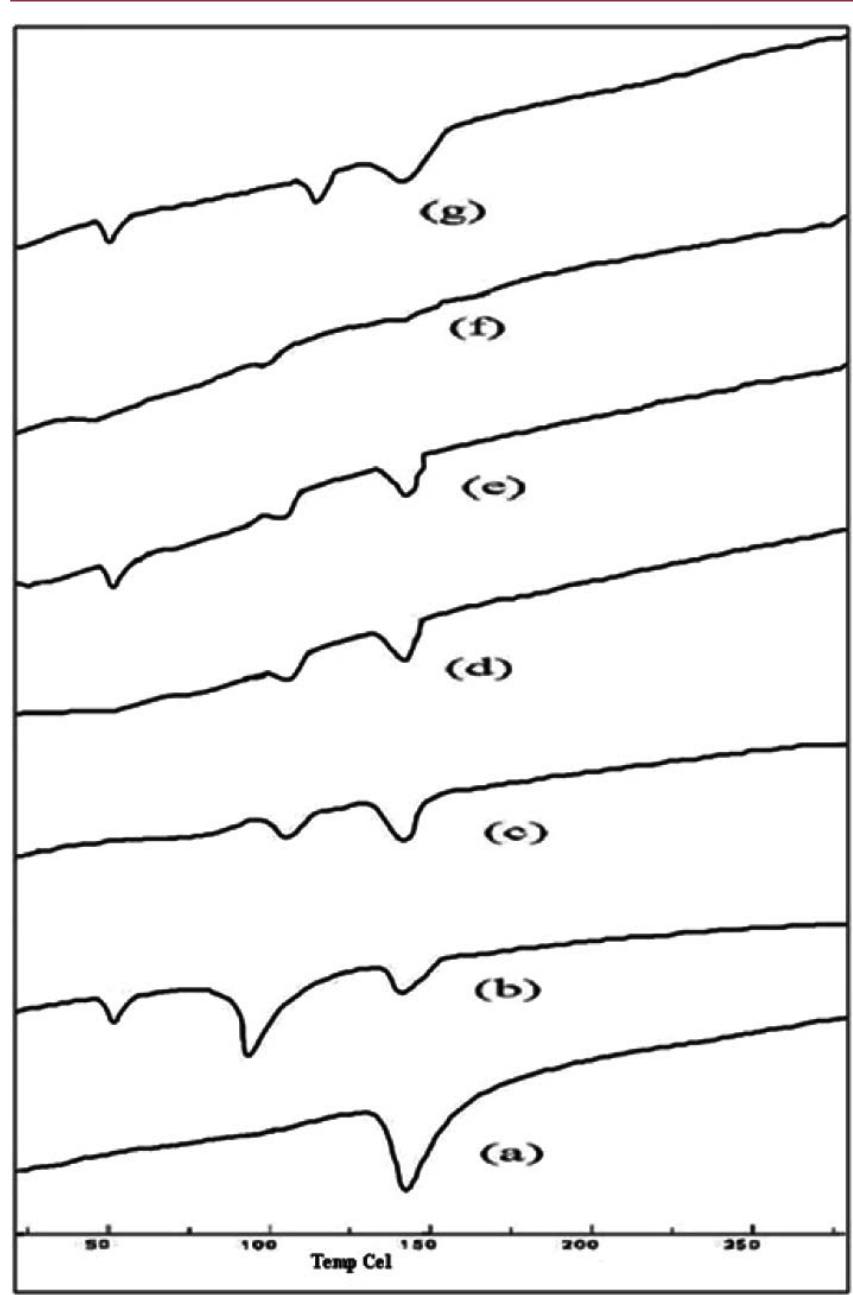

Figure 5. DSC thermograms of (a) ETX, (b) SF4, (c) SF5, (d) SF6, (e) SF7, (f) SF8, and (g) SF12.

agents in the stabilization of sorbitol dispersions. Other factors such as improved glass transition temperature $\left(T_{\mathrm{g}}\right)$ of aged dispersions, decreased crystallinity, and increased wetting of the particles can also be contributors for stabilization.

As understood, a similarity factor greater than 50 indicates similarity in dissolution profiles, thus the aged dispersion SF8 had a dissolution profile similar to fresh dispersion F8, because the amorphization of the fresh dispersion F8 reverted least in the case of SF8. Any change in crystallinity was analyzed by calculation of degree of crystallinity $\left(D_{\text {crys }}\right)$ from DSC thermograms (Figure 5). The $\Delta H_{\text {sd }}$ values and fraction of drug values were used to calculate $D_{\text {crys }}$. In the group of formulations SF5-SF8, the highest $D_{\text {crys }}$ was 8.66 for SF5 (binary dispersion), followed by SF6 and SF7 (ternary dispersions), and was lowest for SF8 (Table 4). Consequently, the amorphous fraction was documented as $99.32 \%$ for SF8. This clearly demonstrates the role of antiplasticizing agents in reducing crystallization of amorphous sugar dispersions. Further investigations are required for studying the effect of varying concentration 


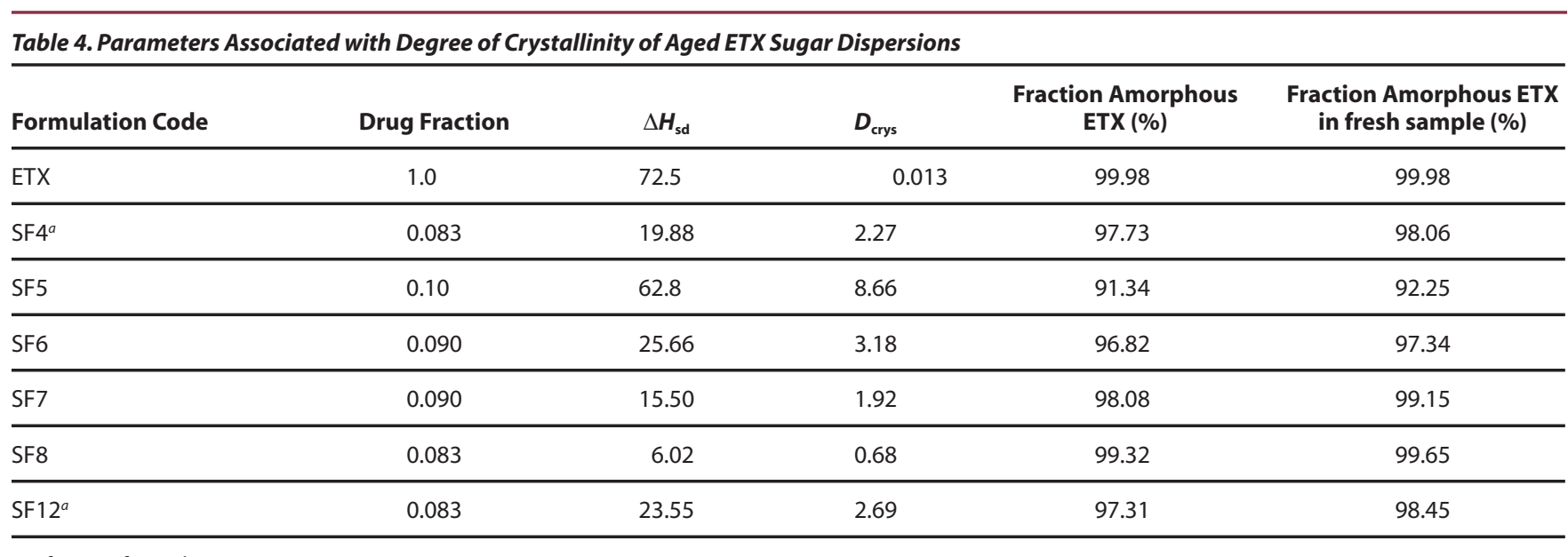

aReference formulations

and type of antiplasticizing agents before any conclusion is affected. However, spectral studies were carried out to get an insight of the physicochemical changes that could help identify the stabilization effect of the antiplasticizing agents.

\section{Visualization and Characterization of ETX Sugar Dispersions}

Scanning electron microscope images of the drug ETX (Figure 6A) show a mixture of plate-like crystals with microparticles, which might have been generated by milling or other size-reduction process at the time of manufacturing.

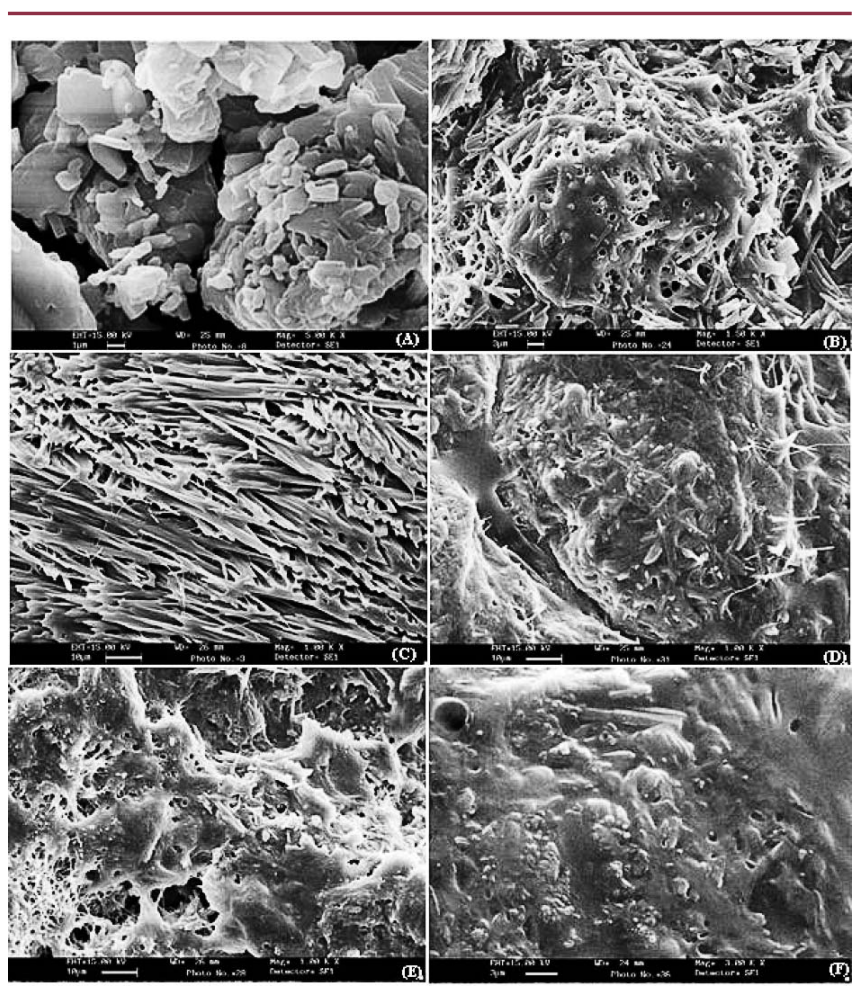

Figure 6. Scanning electron micrographs of $(A) E T X,(B)$ SF5, (C) sorbitol, (D) SF6, (E) SF7, and (F) SF8.

Dissolution Technologies | AUGUST 2011
The microphotograph of SF5 (Figure 6B) shows a partially fused system of ETX and sorbitol with remnants of needleshaped crystals of sorbitol (Figure 6C) that formed as the molten mass cooled, forming a discontinuous solid solution. SEM photomicrographs of SF6 (Figure 6D) and SF7 (Figure 6E) show further loss of sorbitol crystals due to enhancement in miscibility of sorbitol in the presence with PVP K30 and poloxamer 407, respectively. Thus discontinuity of the solid solution was reduced in the presence of the additives, but either the amount of additive incorporated individually was insufficient or a single antiplasticizing agent could not form a continuous solid solution. On the other hand, the micrograph of SF8 containing both PVP K30 and poloxamer 407 shows the complete miscibility of ETX in the quaternary sorbitol dispersion, probably due to formation of glass solution where the matrix is in an amorphous state and the drug is molecularly dispersed throughout the matrix. Thus, the probable cause of the thermodynamic stabilization by antiplasticizing agents is retention of the homogenous amorphous character of the dispersion upon aging.

In addition to visualization, any potential chemical changes that might have occurred during processing and aging were evaluated by analysis of diffuse reflectance spectrographs. The DR spectrum of pure ETX (Figure 7) shows characteristic peaks at $1596.9 \mathrm{~cm}^{-1}(\mathrm{C}=\mathrm{N}$ stretching vibration); $1431 \mathrm{~cm}^{-1}, 1300 \mathrm{~cm}^{-1}, 1141.8 \mathrm{~cm}^{-1}$, and $1085.8 \mathrm{~cm}^{-1}$ ( $\mathrm{S}=\mathrm{O}$ stretching vibrations); and $840.9 \mathrm{~cm}^{-1}$, $775.3 \mathrm{~cm}^{-1}$, and $638 \mathrm{~cm}^{-1}$ (C-Cl stretching vibrations). The spectrum of the physical mixture PMF8 is equivalent to the spectra obtained by the addition of sorbitol, povidone, and poloxamer 407. All characteristic peaks of ETX are retained in the DR spectra of SF5, SF6, SF7, and SF8 dispersions at the same wavenumbers, indicating no modification or interaction between drug and carriers upon aging. The DR spectrum of SF8 dispersion shows sharp peaks at $3680,3150,3826$, and $3548 \mathrm{~cm}^{-1}$ that are not identifiable in the spectra of SF5, SF6, and SF7. However, these peaks are clearly observed in the DR spectrum of PMF8, confirming the stabilization offered by PVP K30 and poloxamer 407. 


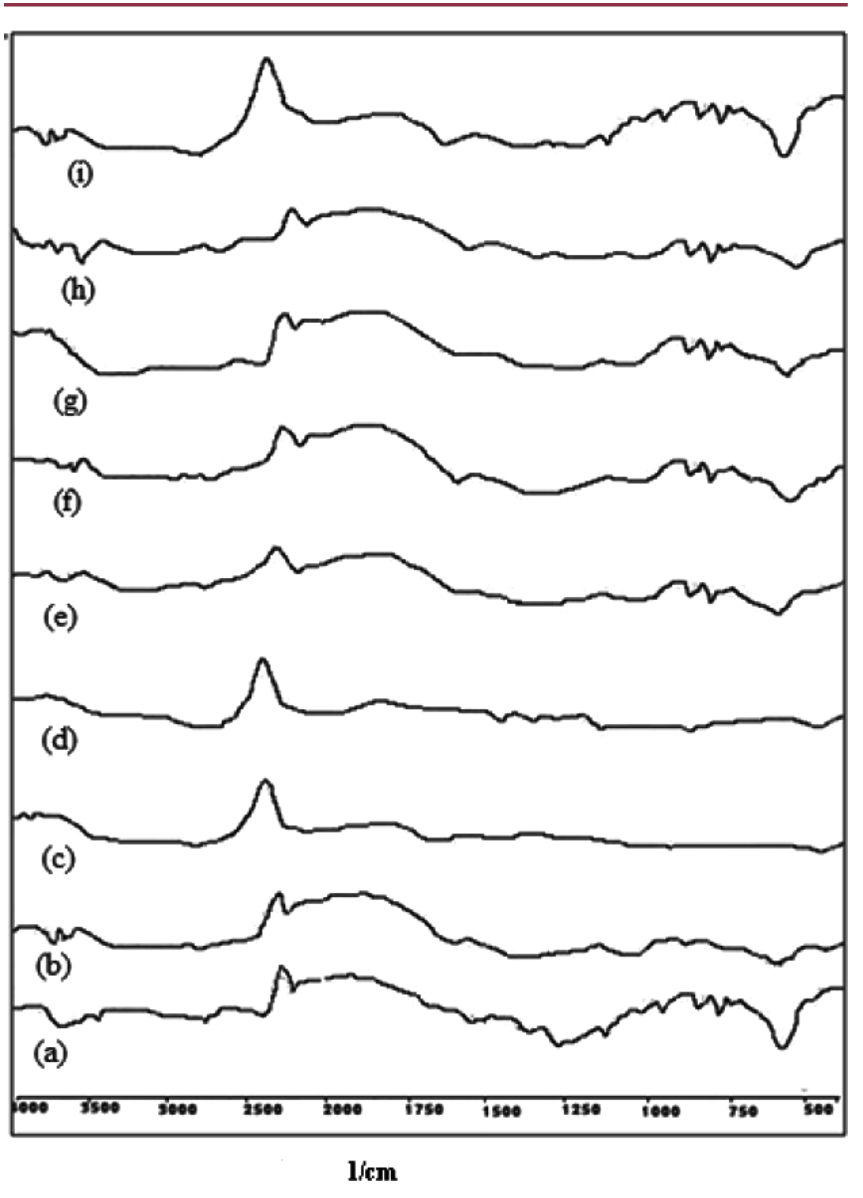

Figure 7. DR spectra of (a) ETX, (b) sorbitol, (c) PVP K30, (d) Poloxamer 407, (e) SF5, (f) SF6, (g) SF7, (h) SF8, and (i) Physical Mixture (PMF8).

\section{CONCLUSION}

This investigation was undertaken to develop stabilized sugar dispersions of etoricoxib and primarily resulted in optimizing the ETX sorbitol-polymer surfactant dispersion that displayed highest dissolution efficiency and was thermodynamically stable. Upon storage, the system underwent the least transformation from amorphous to crystalline form, and hence the dissolution characteristics were not affected significantly. However, more investigation with other antiplasticizing agents is required to achieve higher thermodynamic stability of the sugar dispersions than reported in this study.

\section{ACKNOWLEDGMENT}

The authors acknowledge the SEM and DSC facilities provided by Indian Institute of Technology, Roorkee, India.

\section{REFERENCES}

1. Vasconcelos, T.; Sarmento, B.; Costa, P. Solid dispersions as strategy to improve oral bioavailability of poorly water soluble drugs. Drug Discov. Today. 2007, 12, 1068-1075.
2. Leuner, C.; Dressman, J. Improving drug solubility for oral delivery using solid dispersions. Eur. J. Pharm. Biopharm. 2000, 50, 47-60.

3. Tanaka, N.; Imai, K.; Ueda, S.; Kimura, T. Development of novel sustained-release system, disintegrationcontrolled matrix tablet (DCMT) with solid dispersion granules of nilcadipine (II): in vivo evaluation. J. Controlled Release 2006, 122, 51-56.

4. Water-Insoluble Drug Formulation; Liu, R., Ed.; Interpharm Press: Boca Raton, FL, 2000.

5. Stupak, E. I.; Bates, T.R. Enhanced absorption and dissolution of reserpine-polyvinylpyrrolidone coprecipitates. J. Pharm. Sci. 1972, 61, 400-404.

6. Stupak, E. I.; Bates, T. R. Enhanced absorption of digitoxin from orally administered digitoxinpolyvinylpyrrolidone coprecipitates, J. Pharm. Sci. 1973, 62, 1806-1809.

7. Sekigushi, K.;Obi, N. Studies on absorption of eutectic mixtures. I. A comparison of the behavior of eutectic mixture of sulfathiazole and that of ordinary sulfathiazole in man. Chem. Pharm. Bull. 1961, 9, 866-872.

8. Chiou, W. L.; Riegelman, S. Pharmaceutical applications of solid dispersion systems. J. Pharm. Sci. 1971, 60 (9), 1281-1302.

9. Ozeki, T.; Yuasa, H.; Kanaya, Y. Application of the solid dispersion method to the controlled release of medicine. IX. Difference in the release of flurbiprofen from solid dispersions with poly(ethylene oxide) and hydroxypropylcellulose and the interaction between medicine and polymers. Int. J. Pharm. 1997, 155 (2), 209-217.

10. Ford, J. L. The current status of solid dispersions. Pharm. Acta Helv. 1986, 61, 69-88.

11. Hancock, B.C.; Zografi, G. Characteristics and significance of the amorphous state in pharmaceutical systems. J. Pharm. Sci. 1997, 86 (1), 1-12.

12. Attia, M. A.; Habib, F. S. Dissolution rates of carbamazepine and nitrazepam utilizing sugar solid dispersion system. Drug Dev. Ind. Pharm. 1985, 11, 1957-1969.

13. Van Drooge, D. J.; Hinrichs, W. L.; Frijlink, H.W. Anomalous dissolution behaviour of tablets prepared from sugar glass-based solid dispersions. J. Controlled Release 2004, 97 (3), 441-52.

14. Arias, M. J.; Gines, J. M.; Moyano, J. R.; Rabasco, A. M. The application of solid dispersion technique with D-mannitol to the improvement in oral absorption of triamterene. J.Drug Target. 1994, 2 (1), 45-51.

15. Obikili, A.; Deyme, M.; Wouessidjewe, D.; Duchene, D. Improvement of aqueous solubility and dissolution kinetics of canrenone by solid dispersion in sucroester. Drug Dev. Ind. Pharm. 1998, 14 (6), 791-803.

16. Allen, L. V.; Levinson, R. S.; Martono, D. D. Dissolution rates of corticosteroids utilizing sugar glass dispersions. J. Pharm. Sci. 2006, 66 (4), 494-497.

Dissolution Technologies | AUGUST 2011 
17. Chauhan, B.; Shimpi, S.; Paradkar, A. Preparation and evaluation of glibenclamide-polyglycolized glycerides solid dispersions with silicon dioxide by spray drying technique. Eur. J.Pharm. Sci. 2005, 26, 219-230.

18. Ekelund, K; Osth, K; Pahlstorp, C; Bjork, E; Ulvenlund, S; Johansson, F. Correlation between epithelial toxicity and surfactant structure as derived from the effects of polyethyleneoxide surfactants on caco-2 cell monolayers and pig nasal mucosa.J.Pharm. Sci. 2005, 94 (4), 730-744.

19. Costa, P.; Lobo, J. M. Modeling and comparison of dissolution profiles. Eur. J. Pharm. Sci. 2001, 13, 123-133.

20. Goikhman, A. S.; Irkeli, V. M.; Vavrinyuk, O. S.; Pirogov, U.I.X-ray diffraction determination of the degree of crystallinity of cellulose using a computer. Fibre Chem. 1992, 24 (1), 80-85.

21. Mooter, G. V.D.; Wuyts, M.; Blaton, N.; Grobet, P. Physical stabilization of amorphous ketoconazole in solid dispersions with polyvinylpyrrolidone K25. Eur. J. Pharm. Sci. 2001, 12, 261-269.
22. Patel, H. R.; Patel, R. P.; Patel, M. M. Poloxamers: A pharmaceutical excipients with therapeutic behaviors. Int. J.Pharm. Tech. Res. 2009, 1, 299-303.

23. Shinde, V.R.; Shelake, M. R.; Shetty, S.; Chavanpatil, A. B.; Pore, Y. V.; Late, S. G. Enhanced solubility and dissolution of lamotrigine by inclusion complexation and solid dispersion technique. J. Pharm. Pharmacol. 2008, 60, 1121-1129.

24. Damian, F.; Blaton, B.; Naesens, I.; Balzarini, J.; Kinget, R. Physicochemical characterization of solid dispersions of the antiviral agent UC-781 with polyethylene glycol 6000 and Gelucire 44/14. Eur. J. Pharm. Sci. 2000, 10, 311-322.

25. Waard, H.; Hinrichs, W. L. J.;Visser, M. R. Unexpected differences in dissolution behavior of tablets prepared from solid dispersions with a surfactant physically mixed or incorporated. Int. J. Pharm. 2008, 349, 66-73.

26. Suhagia, B.; Patel, H.; Shah, S. Preparation and characterization of etoricoxib-polyethylene glycol 4000 plus polyvinylpyrrolidone K30 solid dispersions. Acta Pharm. 2006, 56, 285-298. 